\title{
110. On Determination of the Class of Saturation in the Theory of Approximation of Functions
}

\author{
By Gen-ichiro Sunouchi and Chinami WataRI \\ (Comm. by K. KunUGi, M.J.A., Oct. 13, 1958)
}

1. Introduction. Let $f(x)$ be an integrable function, with period $2 \pi$ and let its Fourier series be

$$
\frac{a_{0}}{2}+\sum_{k=1}^{\infty}\left(a_{k} \cos k x+b_{k} \sin k x\right) \equiv \sum_{k=0}^{\infty} A_{k}(x) .
$$

Let $g_{k}(n) k=1,2, \cdots$ be the summating function and consider a family of transforms of (1) of a summability method $G$,

$$
P_{n}(x)=\frac{a_{0}}{2}+\sum_{k=1}^{\infty} g_{k}(n)\left(a_{k} \cos k x+b_{k} \sin k x\right)
$$

where the parameter $n$ needs not be discrete.

If there are a positive non-increasing function $\varphi(n)$ and a class $K$ of functions in such a way that

$$
\begin{array}{lll}
\left\|f(x)-P_{n}(x)\right\|=o(\varphi(n))^{1)} & \text { implies } & f(x)=\text { constant; } \\
\left\|f(x)-P_{n}(x)\right\|=O(\varphi(n)) & \text { implies } & f(x) \in K
\end{array}
$$

(III) for every $f(x) \in K$, one has $\left\|f(x)-P_{n}(x)\right\|=O(\varphi(n))$, then it is said that the method of summation $G$ is saturated with order $\varphi(n)$ and its class of saturation is $K$. This definition is due to J. Favard [2].

The purpose of this article is to determine the order and the class of saturation for several familiar summation methods. M. Zamansky [5] has solved this problem for the method of CesàroFejér, with respect to the space $(C)$ of continuous functions; P. L. Butzer [1] studied the cases of methods of Abel-Poisson and GaussWeierstrass, employing the theory of semi-groups, but, as he made use of the regularity of the spaces $\left(L^{p}\right) p>1$, he left the question open for the spaces $(C)$ and $(L)$.

We give here a direct method to determine the class of saturation for general method of summability, with respect to the spaces $(C)$ and $\left(L^{p}\right) p \geqq 1$. The above condition (I) is easily verified and the condition (III) is proved by so-called singular integral method. The inverse problem (II) is the key point of this paper.

2. The inverse problem. Let us write $\Delta_{n}(x)=f(x)-P_{n}(x)$ and suppose that there are positive constants $c, r$ and $\rho$ such that

$$
\lim _{n \rightarrow \infty} n^{r}\left(1-g_{k}(n)\right)=c k^{\rho 2} \quad(k=1,2, \cdots) .
$$

1) The norm means $(C)$ - or $\left(L^{p}\right)-(p \geqq 1)$ norm.

2) To fix the ideas, we take the limit as $n \rightarrow \infty$; but, as is easily seen, the following arguments remain valid, with appropriate modifications, in other cases (see Theorem 2 below). 
(i) If $\left\|\Delta_{n}(x)\right\|=o\left(n^{-r}\right)$, then we have

$$
a_{k}\left(1-g_{k}(n)\right)=\frac{1}{\pi} \int_{-\pi}^{\pi} \Delta_{n}(x) \cos k x d x=o\left(n^{-r}\right),(k=1,2, \cdots)
$$

and, comparing this with (3), we see

$$
a_{k}=0 \text { and similarly } b_{k}=0 \quad(k=1,2, \cdots)
$$

and consequently we have $f(x)=a_{0} / 2$. Thus the condition (I) is verified.

(ii) Suppose now $\left\|\Delta_{n}(x)\right\|=O\left(n^{-r}\right)$ and let $N<n$. Taking the $N$-th arithmetic mean $\sigma_{N}\left[x ; \Delta_{n}\right]$ of the series

we have

$$
\Delta_{n}(x) \sim \sum_{k=1}^{\infty}\left(1-g_{k}(n)\right) A_{k}(x)
$$

$$
\sigma_{N}\left[x ; \Delta_{n}\right]=\sum_{k=1}^{N}\left(1-g_{k}(n)\right) A_{k}(x)\left(1-\frac{k}{N+1}\right) .
$$

Because it is well known that $\left\|\sigma_{N}[x ; F]\right\| \leqq\|F\|$ (for the spaces $(C)$ and $(L)$, this is trivial; for $\left(L^{p}\right) p>1$, we have only to apply Jensen's inequality), our hypothesis on $\Delta_{n}(x)$ yields

in other words

$$
\left\|\sum_{k=1}^{N}\left(1-g_{k}(n)\right) A_{k}(x)\left(1-\frac{k}{N+1}\right)\right\|=O\left(\frac{1}{n^{r}}\right)
$$

$$
\left\|\sum_{k=1}^{N} n^{r}\left(1-g_{k}(n)\right) A_{k}(x)\left(1-\frac{k}{N+1}\right)\right\|=O(1)
$$

from which it results that, evidently for the space $(C)$ and by means of Fatou's lemma for $\left(L^{p}\right) p \geqq 1$,

$$
\left\|\sum_{k=1}^{N} \lim _{n \rightarrow \infty} n^{r}\left(1-g_{k}(n)\right) A_{k}(x)\left(1-\frac{k}{N+1}\right)\right\|=O(1)
$$

that is to say

$$
\left\|\sum_{k=1}^{N} k^{\rho} A_{k}(x)\left(1-\frac{k}{N+1}\right)\right\|=O(1) .
$$

Denoting by $f^{[\rho]}(x)$ the trigonometric series $\sum_{k=1}^{\infty} k^{\rho} A_{k}(x)$, we see that this is nothing but $\left\|\sigma_{N}\left[x ; f^{[\rho]}\right]\right\|=O(1)$, and the latter is equivalent respectively to

$f^{[\rho]}(x)$ is the Fourier series of a bounded function (for the space $(C)$ ) $f^{[\rho]}(x)$ is the Fourier series of a function in $\left(L^{p}\right)$

(for the space $\left(L^{p}\right), p>1$ )

$f^{[\rho]}(x)$ is the Fourier-Stieltjes series of a function of bounded variation

See for example $[7, \S \S 4,31-4,33]$.

(for the space $(L)$ ).

\section{The method of Cesàro-Fejér summation}

In this case we have

$$
P_{n}(x)=\sum_{k=0}^{n}\left(1-\frac{k}{n+1}\right) A_{k}(x)=\frac{1}{2(n+1) \pi} \int_{-\pi}^{\pi} f(x+t)\left\{\frac{\sin (n+1) t / 2}{\sin t / 2}\right\}^{2} d t
$$

and 


$$
g_{k}(n)=\left(1-\frac{k}{n+1}\right), \quad \lim _{n \rightarrow \infty} n\left(1-g_{k}(n)\right)=k .
$$

The considerations of the preceding section yield

(i) if $\left\|\Delta_{n}(x)\right\|=o(1 / n)$, we have $f(x)=$ constant;

(ii) if $\left\|\Delta_{n}(x)\right\|=O(1 / n)$, we have

$$
\begin{aligned}
& \tilde{f}^{\prime}(x) \in B \text { i.e. } \tilde{f}(x) \in \operatorname{Lip} 1 \quad \text { (for the space }(C) \text { ) } \\
& \tilde{f}^{\prime}(x) \in L^{p} \text { i.e. } \widetilde{f}(x) \in \operatorname{Lip}(1, p) \quad \text { (for the space }\left(L^{p}\right), p>1 \text { ) } \\
& \tilde{f}^{\prime}(x) \in S \text { i.e. } \widetilde{f}(x) \in B V \quad \text { (for the space }(L) \text { ) }
\end{aligned}
$$

respectively. The inverse is known to be true, see A. Zygmund [6]. Thus we have

Theorem 1. The method of Cesàro-Fejér summation is saturated; its order of saturation is $n^{-1}$, its class of saturation is the class of functions $f(x)$ for which

$$
\begin{aligned}
& \tilde{f}(x) \in \operatorname{Lip} 1 \quad \text { (for the space }(C) \text { ) } \\
& \tilde{f}^{\prime}(x) \in L^{p} \quad \text { or } \quad f^{\prime}(x) \in L^{p} \quad \text { (for the space }\left(L^{p}\right), \infty>p>1 \text { ) } \\
& \widetilde{f}(x) \in B V \quad \text { (for the space }(L) \text { ), }
\end{aligned}
$$

respectively.

In a manner similar to that in which we have proved the above theorem, we may show the following theorems.

The Abel-Poisson mean of $\subseteq[f]$ is

$$
P_{r}(x)=\sum_{k=0}^{\infty} A_{k}(x) r^{k}=\frac{1}{2 \pi} \int_{-\pi}^{\pi} f(x+t) \frac{\left(1-r^{2}\right)}{1-2 r \cos t+r^{2}} d t \quad(0 \leqq r<1)
$$

and $g_{k}(r)=r^{k}$. Thus we have

Theorem 2. The method of Abel-Poisson summability is saturated; its order of saturation is $(1-r)$, its class of saturation is identical with that of the method of Cesàro-Fejér summability.

The Riesz mean $\left(R, n^{\rho}, \lambda\right)$ of $\subseteq[f]$ is

$$
R_{n}(x)=\sum_{k=0}^{n}\left(1-\left(\frac{k}{n}\right)^{\rho}\right)^{\lambda} A_{k}(x) \quad \text { and } \quad g_{k}(n)=\left(1-\left(\frac{k}{n}\right)^{\rho}\right)^{\lambda} \text {. }
$$

Theorem 3. The method of Riesz summability $\left(R, n^{\rho}, \lambda\right)$ is saturated; its order of saturation is $n^{-\rho}$, its class of saturation is the class of functions $f(x)$ for which

$$
\begin{array}{ll}
f^{[\rho]}(x) \in B & \text { (for the space }(C)) \\
f^{[\rho]}(x) \in L^{p} & \text { (for the space } \left.\left(L^{p}\right), 1<p<\infty\right) \\
f^{[\rho]}(x) \in S & \text { (for the space }(L))
\end{array}
$$

where $f^{[\rho]}(x)$ denotes the trigonometric series $\sum_{k=1}^{\infty} k^{\rho} A_{k}(x)$.

Corollary. If $\rho$ is a positive integer, the class of saturation of the method of Riesz summability $\left(R, n^{\rho}, \lambda\right)$ is the class of those functions $f(x)$ for which

$$
\begin{array}{ll}
f^{(\rho-1)}(x) \in \operatorname{Lip} 1 & \text { if } \rho \text { is even } \\
\widetilde{f}^{(\rho-1)}(x) \in \operatorname{Lip} 1 & \text { if } \rho \text { is odd }
\end{array} \quad \text { (for the space }(C) \text { ) }
$$




$$
\begin{array}{lll}
f^{(\rho)}(x) \in L^{p} & \left.\quad \text { (for the space }\left(L^{p}\right), 1<p<\infty\right) \text { ) } \\
f^{(\rho-1)}(x) \in B V & \text { if } \rho \text { is even } & \text { (for the space }(L)) . \\
\widetilde{f}^{(\rho-1)}(x) \in B V & \text { if } \rho \text { is odd } &
\end{array}
$$

The Gauss-Weierstrass integral of $f(x)$ is

and $g_{k}(\xi)=e^{-k^{2} \xi / 4}$

$$
W(x ; \xi)=\sum_{n=0}^{\infty} e^{-k^{2} \xi / 4} A_{k}(x)=\sqrt{\frac{\pi}{\xi}} \int_{-\pi}^{\pi} f(x+t) e^{-t^{2} / \xi} d t
$$

Theorem 4. The method of approximation by the Gauss-Weierstrass integral is saturated; its order of saturation is $\xi$; its class of saturation is the class of functions $f(x)$ for which

$$
\begin{array}{ll}
f^{\prime}(x) \in \operatorname{Lip} 1 & \text { (for the space }(C)) \\
f^{\prime \prime}(x) \in L^{p} & \text { (for the space } \left.\left(L^{p}\right), 1<p<\infty\right) \\
f^{\prime}(x) \in B V & \text { (for the space }(L))
\end{array}
$$

respectively.

Since the Bernstein-Rogosinski mean of $\mathfrak{S}[f]$ is defined by

$$
\begin{aligned}
B_{n}(x)= & \frac{1}{2}\left\{S_{n}\left(x+\frac{\pi}{2 n+1}\right)+S_{n}\left(x-\frac{\pi}{2 n+1}\right)\right\} \\
& =A_{0}+\sum_{k=1}^{n} \cos \frac{k \pi}{2 n+1} A_{k}(x)
\end{aligned}
$$

we have $g_{k}(n)=\cos \frac{k \pi}{2 n+1}$ and

Theorem 5. The method of approximation by the BernsteinRogosinski mean of $\subseteq[f]$ is saturated; its order of saturation is $n^{-2}$, and its class of saturation is the class of those functions $f(x)$ for which

respectively.

$$
\begin{array}{ll}
f^{\prime}(x) \in \operatorname{Lip} 1 & \text { (for the space }(C)) \\
f^{\prime \prime}(x) \in L^{p} & \text { (for the space } \left.\left(L^{p}\right), 1<p<\infty\right) \\
f^{\prime}(x) \in B V & (\text { for the space }(L))
\end{array}
$$

Since the integral of de la Vallée Poussin is defined by

$$
\begin{aligned}
V_{n}(x)= & \frac{h_{n}}{2 \pi} \int_{-\pi}^{\pi} f(x+t) \cos ^{2 n} \frac{t}{2} d t \\
= & \sum_{k=0}^{n} \frac{(n !)^{2}}{(n-k) !(n+k) !} A_{n}(x) \quad\left(h_{n}=\frac{2 n(2 n-2) \cdots 4 \cdot 2}{(2 n-1)(2 n-3) \cdots 3 \cdot 1}\right), \\
& g_{k}(n)=\frac{(n !)^{2}}{(n-k) !(n+k) !}=1-\frac{k^{2}}{n}+O\left(\frac{1}{n^{2}}\right),
\end{aligned}
$$

we have, as the answer to a problem proposed by P. L. Butzer [1],

Theorem 6. The method of approximation by the integral of de la Vallée Poussin is saturated; its order of saturation is $n^{-1}$, its class of saturation is the class of functions $f(x)$ for which

$$
\begin{array}{ll}
f^{\prime}(x) \in \operatorname{Lip} 1 & \text { (for the space }(C)) \\
f^{\prime \prime}(x) \in L^{p} & \text { (for the space } \left.\left(L^{p}\right), 1<p<\infty\right)
\end{array}
$$


respectively.

$$
f(x) \in B V \quad \text { (for the space }(L))
$$

The integral of Jackson-de la Vallée Poussin is defined by

$$
\begin{aligned}
I_{n}(x) & =\frac{\tau_{4}}{2 \pi} \int_{-\infty}^{\infty} f\left(x+\frac{2 t}{n}\right) \frac{\sin ^{4} t}{t^{4}} d t \quad\left(\tau_{4}=\frac{1}{2 \pi} \int_{-\infty}^{\infty} \frac{\sin ^{4} t}{t^{4}} d t\right) \\
& =\sum_{k=0}^{2 n-1} h\left(\frac{k}{n}\right) A_{k}(x)
\end{aligned}
$$

where

$$
h(x)= \begin{cases}1-\frac{3}{2} x^{2}+\frac{3}{4}|x|^{3} & |x| \leqq 1 \\ \frac{1}{4}(2-|x|)^{3} & 1 \leqq|x| \leqq 2 \\ 0 & |x| \geqq 2 .\end{cases}
$$

Theorem 7. The method of approximation by the Jackson-de la Vallée Poussin integral is saturated; its order of saturation is $n^{-2}$, its class of saturation is the class of function $f(x)$ for which

$$
\begin{array}{ll}
f^{\prime}(x) \in \operatorname{Lip} 1 & \text { (for the space }(C)) \\
f^{\prime \prime}(x) \in L^{p} & \text { (for the space } \left.\left(L^{p}\right), 1<p<\infty\right) \\
f(x) \in B V & \text { (for the space }(L))
\end{array}
$$

respectively.

The detailed proof of these theorems will appear in another periodical.

The problem (III) of these singular integrals are well known (see B. Sz. Nagy [3], I. P. Natanson [4]).

\section{References}

[1] P. L. Butzer: Math. Ann., 133, 410-425 (1957).

[2] J. Favard: Colloques d'Analyse Harmonique Nancy, Paris, 15, 97-110 (1949).

[3] B. Sz. Nagy: Hungarica Acta Math., 1, 1-39 (1948).

[4] I. P. Natanson: Konstruktive Funkionentheorie, Berlin (1955).

[5] M. Zamansky: Ann. Sci. Ecole Normale Sup., 66, 19-93 (1949).

[6] A. Zygmund: Bull. Amer. Math. Soc., 51, 274-278 (1945).

[7] A. Zygmund: Trigonometrical Series, Warszawa (1935). 